\title{
La Reserpina Aumenta la Expresión de BDNF y PCNA, y Disminuye la de Caspasa-3, en Células Intersticiales (Células de Leydig) de Ratas
}

\author{
Reserpine Increases BDNF and PCNA Expression, but \\ Decreases Caspase-3, in Rat Interstitial Cells (Leydig Cells)
}

Paul Ruiz ${ }^{1-3}$; Patricia Genovese ${ }^{2}$; Alejandro Bielli² ${ }^{2}$ Emiliano Herrera ${ }^{2}$ \& Ricardo Marcos Pautassi ${ }^{3}$

RUIZ, P.; GENOVESE, P.; BIELLI, A.; HERRERA, E. \& PAUTASSI, R. M. La reserpina aumenta la expresión de BDNF y PCNA, y disminuye la de caspasa-3, en células intersticiales (células de Leydig) de ratas. Int. J. Morphol., 36(3):895-900, 2018.

RESUMEN: La reserpina es un antipsicótico e hipotensor arterial que reduce significativamente los niveles de monoaminas centrales, y también es utilizada para modelar los cuadros depresivos humanos en animales de laboratorio. Este trabajo estudió, en ratas Wistar machos adolescentes, cómo la reserpina afecta indicadores moleculares de la función testicular, la cual se ha visto alterada en humanos deprimidos. Una semana luego de finalizado el tratamiento con reserpina ( 4 dosis de 0,0 o $1,0 \mathrm{mg} / \mathrm{Kg}$, cada 2 días) la respuesta ansiosa y depresiva fue evaluada en un laberinto en cruz elevado. Posteriormente, se sacrificaron los animales y disecaron los testículos, los cuales fueron fijados e incluidos en bloques de parafina de donde se obtuvieron cortes histológicos de $6 \mu \mathrm{m}$ de espesor. Estos se utilizaron para medir el diámetro de los túbulos seminíferos y para medir por inmunohistoquímica el porcentaje de células intersticiales (células de Leydig) positivas a (1) Factor neurotrófico derivado del cerebro, (2) antígeno nuclear de células en proliferación (BDNF y PCNA, respectivamente, por sus siglas en inglés), y a (3) caspasa-3. Se obtuvo también un índice de positividad al receptor de andrógenos en las células intersticiales. La expresión del receptor de andrógeno fue evaluada utilizando una escala semicuantitativa de escores (0, 1 , 2 y 3) y el resto de las moléculas por presencia o ausencia de expresión de cada antígeno investigado en 300 células por preparado. Los resultados comportamentales indicaron alteraciones en la respuesta de ansiedad y una significativa depresión motora (e.g., mayor latencia en conductas de escape del sector blanco) en los animales tratados con reserpina. No se observaron diferencias en los diámetros de los túbulos seminíferos ni en la expresión del receptor de andrógeno, mientras que sí se encontró mayor proporción de células intersticiales positivas a BDNF y PCNA, y menor proporción de células positivas a caspasa-3, en los animales tratados. Los resultados corroboran la capacidad de la reserpina para reproducir rasgos comportamentales de la depresión. La administración de la droga, sin embargo, no parece reproducir a nivel testicular los efectos deletéreos encontrados en humanos deprimidos, e incluso los resultados sugieren que la reserpina puede mejorar algunos aspectos de la funcionalidad testicular relacionadas con la actividad de las células intersticiales en ratas.

PALABRAS CLAVE: Testículos; Ratas; BDNF, PCNA; Caspasa.

\section{INTRODUCCIÓN}

La reserpina es un depletor de monoaminas que fue originalmente utilizada como antipsicótico e hipotensor arterial (Seeman \& Lee, 1975; Shamon \& Perez, 2009) y es también empleada para modelar los cuadros depresivos humanos en animales de laboratorio (Ruiz et al., 2017). Este modelo cuenta con críticas de constructo en referencia a la utilidad del mismo para estudiar la depresión en humanos (Leite Hunziker \& Pérez-Acosta, 2001). Se ha observado, sin embargo, que ratas tratadas con reserpina exhiben signos y síntomas similares a los exhibidos por humanos diagnosticados con depresión, incluyendo lentificación en locomoción y mayor consumo de alcohol (Khadrawy et al., 2017; Ruiz et al.). Esto fundamenta la utilización de este modelo para el estudio de las dimensiones biológicas y comportamentales de la depresión (Deussing, 2006), así como para entender su patogenia y evaluar posibles trata-

\footnotetext{
${ }^{1}$ Área de Biofísica, Facultad de Veterinaria, Universidad de la República, Uruguay.

2 Área de Histología y Embriología, Facultad de Veterinaria, Universidad de la República, Uruguay.

${ }^{3}$ Instituto de Investigación Médica Mercedes y Martín Ferreyra, (INIMEC-CONICET - Universidad Nacional de Córdoba), Córdoba, Argentina. Financiación: La investigación que da origen a los resultados presentados en esta publicación recibió fondos de la Agencia Nacional de Investigación e Innovación (ANII-Uruguay) bajo el código POS_EXT_2014_1_105 877, de la Comisión Sectorial de Investigación Científica de la Universidad de la República (CSIC-UdelaR) y de la Agencia Nacional de Promoción Científica y Tecnológica de Argentina (ANPCyT), bajo el código PICT 2015-0325.
} 
mientos (Escorihuela \& Fernandez-Terual, 1998). Existen sin embargo variables en las que no se ve analogía entre trastorno y modelo. Por ejemplo, la reserpina aumenta los niveles de hormonas tiroideas, en tanto que en humanos lo más común es que la depresión curse con hipotiroidismo (Ruiz et al.). Se conoce muy poco la manera en que la reserpina afecta otras variables relacionadas con depresión en humanos, tales como la espermatogénesis y los niveles de testosterona, que se encuentran en niveles más bajos en individuos deprimidos (Schweiger et al., 1999; Zarrouf et al., 2009).

Existen numerosos marcadores moleculares que dan cuenta de la función testicular. Uno de ellos es el factor neurotrópico derivado del cerebro (BDNF, por sus siglas en inglés). La concentración local de dicho factor tiene un papel relevante en el desarrollo de la depresión, viéndose alterado en el hipocampo y la corteza pre-frontal. Además, la variación de dichas concentraciones ha sido emparentada con la estabilidad del humor y con otras psicopatologías (Angelucci et al., 2005). El BDNF también se encuentra a nivel testicular (Mutter et al., 1999), particularmente en las células intersticiales (células de Leydig) (Yochkova et al., 2015). Se ha propuesto varias funciones para esta molécula a nivel testicular, relacionadas con la proliferación celular (Yochkova et al.), el desarrollo testicular (Li \& Zhou, 2013) y la espermatogénesis (Safari et al., 2017). A su vez, existen otros marcadores de desarrollo y proliferación celular en los testículos como el antígeno nuclear de células en proliferación (PCNA). Esta es una proteína intranuclear, cofactor de la ADN polimerasa, que también se encuentra en las células intersticiales, entre otros tipos celulares (Chapman \& Wolgemuth, 1994).

Además de moléculas que señalan proliferación celular en los testículos, existen las que marcan muerte celular, como es el caso de la caspasa-3. Esta enzima está asociada a la muerte celular por apoptosis, aumentando su presencia en estos procesos. En el caso de las células intersticiales además de estar asociada a los procesos de muerte celular, está asociada a la disminución de la testosterona (Kim et al., 2001), principal hormona producida por estas células.

Otros indicadores de la función testicular son la abundancia de receptores de andrógenos (RA) y el diámetro de los túbulos seminíferos. Los RA median el efecto de los andrógenos, especialmente de la testosterona, hormona que regula el inicio y mantenimiento de la espermatogénesis en los machos. La ausencia o disminución de la expresión de RA en células intersticiales está asociada con problemas en la espermatogénesis (Lavalle \& Lalosa, 2015). El diámetro de los túbulos seminíferos también está asociado a los niveles de testosterona y sobre todo a la espermatogénesis. La espermatogénesis depende de los niveles intratesticulares de testosterona y de la morfología de los túbulos seminíferos, por lo que mayor diámetro de los túbulos seminíferos implica mayores niveles de testosterona y mayor producción espermatogenética (Rodríguez et al., 1999).

El objetivo de este trabajo fue estudiar si la reserpina, como modelo farmacológico de depresión en animales de laboratorio, reproduce en ratas los efectos deletéreos sobre las células productoras de testosterona que fueran reportados a nivel testicular en humanos deprimidos (Schweiger et al.; Zarrouf et al.). Para esto se trataron ratas macho, adolescentes, con reserpina, empleando una dosis con la que previamente observamos signos de conducta depresiva (Ruiz et al.) y se estudiaron marcadores de la función testicular. Específicamente, se midió mediante inmunohistoquímica la positividad a BDNF, PCNA, caspasa-3 y RA en las células intersticiales, así como el diámetro de los túbulos seminíferos. Para confirmar la efectividad de la reserpina en inducir síntomas depresivos se evaluó a los animales en el laberinto elevado en cruz (LEC).

La decisión de analizar estos fenómenos en ratas adolescentes radica en que en esta etapa se produce un aumento de la producción de testosterona, y el inicio de la espermatogénesis. La adolescencia es, asimismo, una de las etapas vitales más afectadas por la depresión (Ruiz et al.).

\section{MATERIAL Y MÉTODO}

Sujetos, diseño experimental y tratamiento farmacológico. Los animales, 14 ratas Wistar machos de 30 días de edad -adolescentes- al inicio del experimento, fueron provistos por la Unidad de Reactivos Biológicos de Experimentación de la Facultad de Medicina de la Universidad de la República (URBE-Fmed-UdelaR). Los animales fueron mantenidos con agua y comida ad-libitum y fueron administrados con reserpina [0.0 (vehículo) o $1,0 \mathrm{mg} /$ $\mathrm{kg}$, cada 48 horas por 8 días, Sigma-Aldrich, EE.UU.]. La reserpina se vehiculizó en solución fisiológica y se administró de manera intraperitoneal en un volumen de 0,015 $\mathrm{ml} / \mathrm{gr}$ de peso. Veinticuatro horas de finalizado el protocolo se aplicó a las ratas el test del laberinto elevado en cruz y, una semana después, fueron sacrificados y sus testículos pesados y fijados en solución fijadora de Bouin. Se hicieron bloques de parafina y se obtuvieron cortes de 6 micrómetros de espesor que luego fueron teñidos con hematoxilina y eosina o se les aplicó inmunohistoquímica. El protocolo de sacrificio fue aprobado por la Comisión de Ética en Uso de Animales de la Facultad de Veterinaria de Universidad de la República (CEUA-Fvet-UdelaR, Exp nº111130-000658-13), Uruguay. 
Test de Laberinto Elevado en Cruz (LEC). El propósito de esta evaluación fue confirmar la acción depresiva de la reserpina a nivel comportamental. El test de LEC es ampliamente usado para medir respuesta ansiosa (tiempo en brazos) y motora (entradas a brazos), la cual está asociada a la patología depresiva, dado que en la depresión la actividad motora está disminuida (Ruiz et al.). El aparato empleado era una plataforma en forma de cruz, elevada a $50 \mathrm{~cm}$ del suelo. La plataforma estaba compuesta por dos brazos abiertos de 50 x $10 \mathrm{~cm}$ y dos cerrados de $50 \times 10$ con paredes de 40 $\mathrm{cm}$ de altura y descubierta en la parte superior.

En cada sesión experimental los animales se pusieron de manera individual en el centro del laberinto, enfrentando un brazo abierto. Los animales se retiraron del laberinto después de 5 minutos de exploración libre. Las sesiones se realizaron en la tarde (14:00 a 18:00 h) en una habitación iluminada con luz artificial blanca y aislada acústicamente.

Las pruebas se grabaron por medio de una cámara de video ubicada en el techo de la habitación. Posteriormente, los archivos de video fueron evaluados, registrándose los siguientes patrones comportamentales: a) Frecuencia de entradas a brazos abiertos y cerrados; b) tiempo en cada uno de los brazos y en el centro; c) latencia para ingresar a brazos cerrados. Siguiendo estudios previos (Acevedo et al., 2016), la latencia de la primera entrada al brazo cerrado y el tiempo pasado en los brazos abiertos fueron considerados índices de respuesta ansiosa, en tanto que la frecuencia de entradas a los brazos cerrados fue considerado un índice de locomoción.

Inmunohistoquímica e histología cuantitativa. Los testículos fueron disecados, pesados y fijados por inmersión en solución fijadora de Bouin durante 24 horas. Fueron deshidratados por inmersión sucesiva en etanol $70^{\circ}, 95^{\circ}$ y $100^{\circ}$ e incluidos en bloques de parafina. Se obtuvo cortes de $6 \mu \mathrm{m}$ de cada animal teñidos con hematoxilina y eosina para medir el diámetro de los túbulos seminíferos (30/corte). Para los ensayos de inmunohistoquímica, tras colocar los portaobjetos con las secciones de los tejidos en estufa (60 ${ }^{\circ} \mathrm{C}, 10 \mathrm{~min}$ ), las mismas se colocaron en xilol (15 min y luego otros 10 en nuevo xilol). Posteriormente se hidrató las muestras en concentraciones decrecientes de etanol $\left(100^{\circ}\right.$, $95^{\circ}, 70^{\circ}$ ) y se las lavó en PBS (5 min). Luego se recuperó los antígenos con horno microondas, con las secciones sumergidas en solución buffer citrato $0,01 \mathrm{M}, \mathrm{pH} 6,0$, por 3 min, a $100 \%$ de potencia, luego por $12 \mathrm{~min}$ a $40 \%$ de potencia y luego se dejó reposar por $20 \mathrm{~min}$. Se lavó nuevamente en PBS (5 min), se colocó el suero bloqueante y luego el anticuerpo primario: en láminas diferentes, obtenidas de cada animal, se aplicó ya sea anticuerpo primario antiBDNF (Santa Cruz Biotechnology, CA, USA), anti caspasa
3 (AB 4051, Abcam, UK), anti PCNA (Dako M0879, USA) o anticuerpo primario de ratón anti AR humano (AR N-20 SC-816, Santa Cruz, USA) en cámara húmeda durante toda la noche. Se utilizó el mismo kit amplificador Mach 2 Double (Mouse-HRP + Rabbit-AP, Polymer detection kit, Biocare medical, USA) y cromógeno DAB en los cuatro casos. Se agregó un preparado como control negativo para cada ensayo (sin aplicar anticuerpo primarios) y controles positivos para cada uno de los anticuerpos primarios.

La evaluación se hizo capturando imágenes de los preparados por medio de un microscopio óptico Olympus BX50 (Olympus, Tokio, Japón), video cámara digital (Infinity 1; Lumenera, Toronto, ON, Canada), computadora personal con programa Infinity módulos Capture y Analize (Lumenera, Toronto, ON, Canada) para captura y análisis de imágenes, las cuales fueron evaluadas por observadores entrenados. La positividad a PCNA, caspasa-3 y BDNF fue evaluada en 300 células intersticiales por preparado, mientras que para los RA se calculó el índice de positividad para cada preparado considerando un score cualitativo (0: negativo, 1: débil, 2: moderado, 3: intenso). El índice de positividad se calculó como $1 \times n 1+2 \times n 2+3 \times n 3 /$ cantidad de células contadas, donde $\mathrm{n}=$ proporción de células por campo que muestran débil (1), moderada (2) o intensa (3) positividad.

Análisis estadístico. Todas las variables registradas (pesos, variables en el LAC, y aquellas derivadas de la evaluación histofisiológica) fueron analizadas por T de Student para grupos independientes (i.e., tratados vs. controles). Los valores de $\mathrm{p}<0,05$ se consideraron estadísticamente significativos y los resultados se expresan como media \pm SEM.

\section{RESULTADOS}

Laberinto Elevado en Cruz. El test se aplicó el día siguiente a finalizar el protocolo de administración de reserpina. Los resultados fueron coherentes con trabajos previos de nuestro laboratorio (Ruiz et al.) y confirmaron el efecto depresor de reserpina. Los animales tratados con esta droga tuvieron, en comparación con los controles tratados con vehículo, una latencia significativamente mayor $(t=2,15, p<0,05)$ para ingresar a los brazos cerrados, además de tener $(\mathrm{t}=$ $2,21, \mathrm{p}<0,05)$ significativamente menos ingresos a los brazos cerrados (Fig. 1). Estos resultados evidencian una ralentización en la actividad motora y una alteración en la respuesta a situaciones de riesgo, producto de la administración de reserpina, y son similares a los resultados encontrados en trabajos previos de nuestro grupo en el campo abierto y en la prueba de la caja de luz oscuridad (Ruiz et al.). No 
Tabla I. Variables registradas en Laberinto elevado en cruz.

\begin{tabular}{llll}
\hline & Reserpina & Control & $\mathrm{p}<0,05$ \\
\hline Latencia para ingresar a brazos cerrados (segs) & $10,5 \pm 10,5$ & $5,6 \pm 1,3$ & SI \\
Tiempo en brazos abiertos (segs) & $3,7 \pm 2,9$ & $14,7 \pm 11,7$ & NO \\
Tiempo en brazos cerrados (segs) & $252,1 \pm 13,3$ & $264,5 \pm 11,4$ & NO \\
Tiempo en el centro (segs) & $21,3 \pm 11,9$ & $21,0 \pm 7,7$ & NO \\
Frecuencia de entradas en brazos cerrados & $3,6 \pm 1,0$ & $6,8 \pm 1,1$ & SI \\
Frecuencia de entradas en brazos abiertos & $0,4 \pm 0,3$ & $1,8 \pm 0,7$ & NO
\end{tabular}

Medias y errores para las variables estudiadas en animales tratados con reserpina y controles. Se indica para cuales variables se encontraron diferencias significativas $(\mathrm{p}<0,05)$ entre grupos.
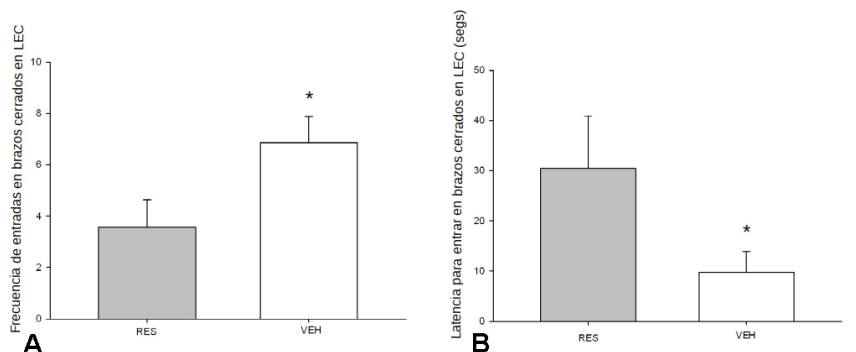

Fig. 1. Respuestas de locomoción y ansiedad en el laberinto elevado en cruz (LEC), en ratas adolescentes tratadas con reserpina o vehículo. Los animales tratados con reserpina (RES) exhibieron, en comparación con sus pares tratados con vehículo (VEH), significativamente menos ingresos a los brazos cerrados (Panel A) y una mayor latencia para ingresar por primera vez a los brazos cerrados (Panel B). Los valores expresan las medias \pm desvío estándar, y los asteriscos denotan diferencias según test $\mathrm{T}$ de Student $(\mathrm{p}<0,05)$.
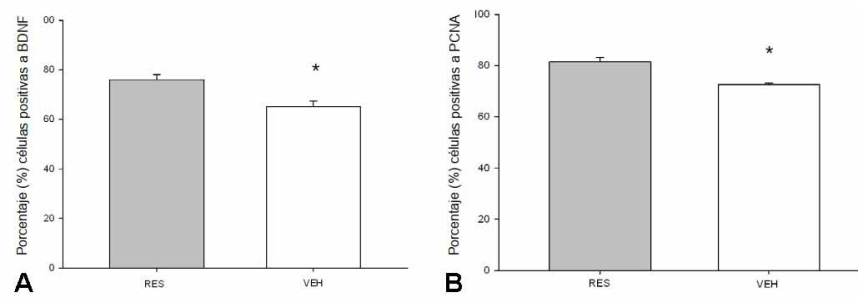

se encontraron diferencias significativas entre los grupos en el resto de las variables (i.e., tiempo en brazos abiertos y cerrados, ingresos a brazos abiertos) (Tabla I).

Histofisiología testicular. No se encontraron diferencias inherentes al tratamiento en el peso de los animales, ni en el peso de los testículos promediados, ni entre sí (derecho vs derecho, izquierdo vs izquierdo) (Tabla I). A nivel molecular, se encontraron diferencias significativas en la positividad de las células intersticiales a BDNF y PCNA (Fig. 2). Los animales tratados con reserpina expresaron significativas mayor proporción de células positivas a ambas proteínas que sus pares controles $[(\mathrm{t}=3,59, \mathrm{p}<0,05)$ y $(\mathrm{t}=4,78, \mathrm{p}<0,001)$; BDNF y PCNA, respectivamente]. Por el contrario, los animales tratados expresaron significativamente menos $(\mathrm{t}=-2,84$, $\mathrm{p}<0,01)$ proporción de células positivas a caspasa-3 que sus pares controles (Fig. 2).

En tanto, no se encontraron diferencias significativas entre grupos en el índice de positividad a RA en las células intersticiales ni en los diámetros de los túbulos seminíferos (Tabla II).

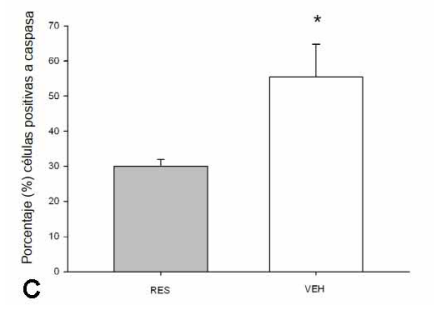

Fig. 2. Inmunoreactividad para BDNF, PCNA y caspasa-3 en células intersticiales, en animales tratados con reserpina (RES) y en controles tratados con vehículo (VEH). Los animales del grupo RES expresaron significativamente mayor porcentaje de positividad a BDNF (A) y PCNA (B), pero significativamente menos porcentaje de caspasa-3 (C), que sus pares controles. Los valores expresan las medias \pm desvío estándar, y los asteriscos denotan diferencias estadísticamente significativas según test T de Student $(\mathrm{p}<0,05)$.

Tabla II. Variables histofisiológicas registradas.

\begin{tabular}{llll}
\hline & Reserpina & Control & $\mathrm{p}<0,05$ \\
\hline Peso al sacrificio (gramos) & $189,6 \pm 7,9$ & $177,1 \pm 15,7$ & NO \\
Peso testículos (promedio en gramos) & $1,13 \pm 0,04$ & $1,72 \pm 0,63$ & NO \\
Porcentaje (\%) células de Leydig + a BDNF & $76,0 \pm 2,0$ & $65,1 \pm 2,2$ & SI \\
Porcentaje (\%) células de Leydig + a caspasa-3 & $30,0 \pm 1,9$ & $55,4 \pm 9,4$ & SI \\
Porcentaje (\%) células de Leydig + a PCNA & $81,4 \pm 1,7$ & $72,5 \pm 1,7$ & SI \\
Indice de positividad en células de Leydig a RA & $1,1 \pm 0,07$ & $1,3 \pm 0,02$ & NO \\
Diámetro de túbulos seminíferos (micrometros) & $520,4 \pm 17,3$ & $479,5 \pm 12,7$ & NO \\
\hline
\end{tabular}

Medias y errores para las variables estudiadas en animales tratados con reserpina y controles. Se indica para cuales variables se encontraron diferencias significativas $(\mathrm{p}<0,05)$ entre grupos. 


\section{DISCUSIÓN}

Los resultados obtenidos aportan nueva información sobre la validez de la administración de reserpina como modelo farmacológico de depresión, sobre el impacto de esta droga en los testículos y sobre su potencial uso biotecnológico.

En lo que respecta al primer punto, la reserpina ha demostrado ser un modelo farmacológico válido para modelar síntomas depresivos en animales de laboratorio dado que modela muchos síntomas vistos en humanos deprimidos: enlentecimiento motor, baja de niveles de monoaminas centrales y aumento del consumo de alcohol (Khadrawy et al.; Ruiz et al.); sin embargo, hay variables características de la depresión en humanos, como el hipotiroidismo, que la reserpina no reproduce en animales de laboratorio (Ruiz et $a l$. .). Los resultados de este trabajo son consistentes con esta literatura. Por un lado, observamos significativas alteraciones en patrones de ansiedad y actividad locomotora en el LEC en los animales tratados con reserpina. Estos animales se desplazaron menos (menos movimientos entre brazos) y tuvieron una respuesta de ansiedad alterada (mayor latencia en ingreso a brazos cerrados). Sin embargo, los resultados también indicaron que la reserpina mejora la función testicular a nivel de las células intersticiales, dado que aumenta los niveles de BDNF y PCNA asociados con mayores niveles de testosterona (El-Gehani et al., 1998; Jeon et al., 2017). Estos datos contrastan con lo observado en humanos, donde el diagnóstico de depresión está asociado a bajos niveles de testosterona (Schweiger et al.). De hecho, hay protocolos que usan esta hormona para el tratamiento farmacológico de la depresión (Zarrouf et al.).

Los resultados pueden considerarse en el marco del potencial uso biotecnológico de la reserpina, como tratamiento para mejorar la función testicular. Existen escasas investigaciones en animales de laboratorio sobre el efecto de la reserpina en variables reproductivas en macho (Adams \& Fudge, 1959; Thomas et al., 1969), y en su mayoría las mismas subrayan efectos negativos de esta droga. Ninguna de ellas, sin embargo, empleó los indicadores moleculares, o el protocolo utilizado en este trabajo. Muchos trabajos plantean que los factores neurotróficos, dentro de ellos el BDNF, tienen uso potencial como tratamientos para mejorar la espermatogénesis (Mutter et al.; Li \& Zhou; Safari et al.; Yochkova et al.). De igual forma el PCNA está asociado con mejoras en la espermatogénsis (Tousson et al., 2011), y los mayores niveles de caspasa-3 con muerte celular (Shaha et al., 2010). El aumento observado en los marcadores de diferenciación y proliferación celular en células intersticiales, encargadas de producir testosterona y estimular la espermatogénesis, así como la disminución de marcadores de muerte celular, sugiere que la reserpina es una droga con potencial para mejorar la función androgénica testicular. Esta actividad androgénica mejorada, de ocurrir intensamente, podría tener efectos sobre la espermatogénesis. Sin embargo, el diámetro de los túbulos seminíferos indica que no hubo efecto sobre la espermatogénesis. El efecto estimulador sobre el número y actividad de las células intersticiales implica una hipótesis que futuros estudios deberán investigar empleando marcadores moleculares alternativos (i.e. enzimas esteroidogénicas) y diferentes protocolos de administración, así como la determinación de concentraciones séricas de testosterona.

RUIZ, P.; GENOVESE, P.; BIELLI, A.; HERRERA, E. \& PAUTASSI, R. M. Reserpine increases BDNF and PCNA expression, but decreases caspase-3, in rat Interstitial Cells (Leydig cells). Int. J. Morphol., 36(3):895-900, 2018.

SUMMARY: Reserpine, a drug that depletes central monoamines, has been used as an antipsychotic and arterial hypotensive, and to model depression in animals. The present study analyzed, in adolescent male rats, the effects of chronic reserpine treatment on molecular indexes of testicular function. A week after termination of the treatment ( 4 doses of 0,0 or $1,0 \mathrm{mg} / \mathrm{Kg} /$ every $48 \mathrm{~h}$ ) the animals were tested for anxiety response and depression patterns in an elevated plus maze. They were then euthanized, their testes dissected, fixed and embedded in paraffin to obtain blocks. Histological sections $(6 \mu \mathrm{m})$ were obtained and used to measure the diameter of seminiferous tubules and the expression in Leydig cells of Brain-derived neurotrophic factor (BDNF), Proliferating cell nuclear antigen (PCNA), Caspase-3 and androgen receptors, by immunohistochemistry. Behavioral results indicated significant alterations in anxiety responses and a significant motor depression (e.g., greater latency to escape from the white sector). There were no differences between groups in the diameter of seminiferous tubules nor in the androgen receptors positivity. Reserpine-treated animals, however, exhibited more BDNF and PCNA positive cells, and less positive Caspase-3 cells in Leydig cells, than control animals. The results corroborate the efficacy of reserpine to reproduce some of the behavioral components of depression. The drug, however, does not seem to exert in rats the same effects on testicular function that have been found in humans diagnosed with depression. Furthermore the drug seems to enhance some aspects of testicular function related to Leydig cells function in rats.

KEY WORDS: Testicles; Rats; BDNF; PCNA; Caspase.

\section{REFERENCIAS BIBLIOGRÁFICAS}

Acevedo, M. B.; Fabio, M. C.; Fernández, M. S. \& Pautassi, R. M. Anxiety response and restraint-induced stress differentially affect ethanol intake in female adolescent rats. Neuroscience, 334:259-74, 2016.

Adams, A. E. \& Fudge, M. W. Effects of reserpine on the reproductive 
system of immature male mice. J. Exp. Zool., 142(1):337-51, 1959.

Angelucci, F.; Brenè, S. \& Mathé, A. A. BDNF in schizophrenia, depression and corresponding animal models. Mol. Psychiatry, 10(4):345-52, 2005.

Chapman, D. L. \& Wolgemuth, D. J. Expression of proliferating cell nuclear antigen in the mouse germ line and surrounding somatic cells suggests both proliferation-dependent and -independent modes of function. Int. J. Dev. Biol., 38(3):491-7, 1994.

Deussing, J. M. Animal models of depression. Drug Discov. Today Dis. Models, 3(4):375-83, 2006.

El-Gehani, F.; Zhang, F. P.; Pakarinen, P.; Rannikko, A. \& Huhtaniemi, I. Gonadotropin-independent regulation of steroidogenesis in the fetal rat testis. Biol. Reprod., 58(1):116-23, 1998.

Escorihuela, R. \& Fernandez-Terual, A. Modelos animales en psicopatología y psicofarmacología: del análisis experimental de la conducta a la neurogenética. Psicol. Conduc., 6(1):165-91, 1998.

Jeon, W. Y.; Kim, O. S.; Seo, C. S.; Jin, S. E.; Kim, J. A.; Shin, H. K.; Kim, Y. U. \& Lee, M. Y. Inhibitory effects of Ponciri Fructus on testosteroneinduced benign prostatic hyperplasia in rats. B. M. C. Complement. Altern. Med., 17(1):384, 2017.

Khadrawy, Y. A.; Sawie, H. G.; Abdel-Salam, O. M. E. \& Hosny, E. N. Cannabis exacerbates depressive symptoms in rat model induced by reserpine. Behav. Brain Res., 324:41-50, 2017.

Kim, J. M.; Ghosh, S. R.; Weil, A. C. \& Zirkin, B. R. Caspase-3 and caspaseactivated deoxyribonuclease are associated with testicular germ cell apoptosis resulting from reduced intratesticular testosterone. Endocrinology, 142(9):3809-16, 2001.

Lavalle, O. A. \& Lalosa, S. Implicancias fisiopatológicas del receptor androgénico. Mutaciones, polimorfismos y patologías asociadas. Rev. Argent. Endocrinol. Metab., 52(2):79-107, 2015.

Leite Hunziker, M. H. \& Pérez-Acosta, A. M. Modelos animales en psicopatología: ¿una contribución o una ilusión?. Av. Psicol. Clin. Latinoam., 19:37-50, 2001.

$\mathrm{Li}, \mathrm{C}$. \& Zhou, X. The potential roles of neurotrophins in male reproduction. Reproduction, 145(4):R89-95, 2013.

Mutter, D.; Middendorff, R. \& Davidoff, M. S. Neurotrophic factors in the testis. Biomed. Rev., 10:25-30, 1999.

Rodríguez, H.; Salazar, P.; Schmidt, N.; Torres, P. \& Ossandón, E. Human testicular histology in young and senile men. Rev. Chil. Anat., 17(2):1838, 1999.

Ruiz, P.; Calliari, A. \& Pautassi, R. Consumo de alcohol en ratas adolescentes tratadas con reserpina y fluoxetina. Suma Psicol., 24(1):67-77, 2017.

Safari, H.; Khanlarkhani, N.; Sobhani, A.; Najafi, A. \& Amidi, F. Effect of brain-derived neurotrophic factor (BDNF) on sperm quality of normozoospermic men. Hum. Fertil. (Camb.), 5:1-7, 2017.

Schweiger, U.; Deuschle, M.; Weber, B.; Körner, A.; Lammers, C. H.; Schmider, J.; Gotthardt, U. \& Heuser, I. Testosterone, gonadotropin, and cortisol secretion in male patients with major depression. Psychosom. Med., 61(3):292-6, 1999.

Seeman, P. \& Lee, T. Antipsychotic drugs: direct correlation between clinical potency and presynaptic action on dopamine neurons. Science, 188(4194):1217-9, 1975.

Shaha, C.; Tripathi, R. \& Mishra, D. P. Male germ cell apoptosis: regulation and biology. Philos. Trans. R. Soc. Lond. B Biol. Sci., 365(1546):150115, 2010.

Shamon, S. D. \& Perez, M. I. Blood pressure lowering efficacy of reserpine for primary hypertension. Cochrane Database Syst. Rev., 7(4):CD007655, 2009.

Thomas, J. A.; Knych, E. T. Jr. \& Mawhinney, M. G. Effect of reserpine on the uptake of 1,2-3H-testosterone by mouse prostate glands. Eur. J. Pharmacol., 8(3):361-3, 1969.

Tousson, E.; Ali, E. M.; Ibrahim, W. \& Mansour, M. A. Proliferating cell nuclear antigen as a molecular biomarker for spermatogenesis in PTUinduced hypothyroidism of rats. Reprod. Sci., 18(7):679-86, 2011.

Yochkova, S.; Lakova, L.; Pavlova, E.; Dimova, D. \& Atanassova, N. Role of Brain-Derived Neurotrophic Factor (BDNF) and its receptor TrkB in reproductive system in developing rats: possible role for Leydig cell and germ cell development. Am. J. Reprod. Immunol., 73(Suppl. 1):41$2,2015$.

Zarrouf, F. A.; Artz, S.; Griffith, J.; Sirbu, C. \& Kommor, M. Testosterone and depression: systematic review and meta-analysis. J. Psychiatr. Pract., 15(4):289-305, 2009.

\section{Dirección para correspondencia: \\ Paul Ruiz \\ Área de Biofísica \\ Facultad de Veterinaria \\ Universidad de la República \\ URUGUAY}

Email: paulruiz@fvet.edu.uy

Recibido : 09-01-2018

Aceptado: 22-03-2018 